\title{
Functional Weak Laws for the Weighted Mean Losses or Gains and Applications
}

\author{
Gane Samb Lo1,2, Serigne Touba Sall1,3, Pape Djiby Mergane ${ }^{1}$ \\ ${ }^{1}$ LERSTAD, Université Gaston Berger de Saint-Louis, Saint-Louis, Sénégal \\ ${ }^{2}$ LSTA, Université Pierre et Marie Curie, Paris, France \\ ${ }^{3}$ Ecole Normale Supérieure, Dakar, Sénégal \\ Email: pdmergane@ufrsat.org
}

Received 22 February 2015; accepted 24 May 2015; published 27 May 2015

Copyright (@ 2015 by authors and Scientific Research Publishing Inc.

This work is licensed under the Creative Commons Attribution International License (CC BY). http://creativecommons.org/licenses/by/4.0/

(c) (i) Open Access

\section{Abstract}

In this paper, we show that many risk measures arising in Actuarial Sciences, Finance, Medicine, Welfare analysis, etc. are gathered in classes of Weighted Mean Loss or Gain (WMLG) statistics. Some of them are Upper Threshold Based (UTH) or Lower Threshold Based (LTH). These statistics may be time-dependent when the scene is monitored in the time and depend on specific functions $w$ and $d$. This paper provides time-dependent and uniformly functional weak asymptotic laws that allow temporal and spatial studies of the risk as well as comparison among statistics in terms of dependence and mutual influence. The results are particularized for usual statistics like the Kakwani and Shorrocks ones that are mainly used in welfare analysis. Data-driven applications based on pseudo-panel data are provided.

\section{Keywords}

Empirical Process, Time Dependent Process, Weak Theory, Risk Measures, Poverty Index, Loss Function, Economic Welfare

\section{Introduction and Motivation}

In many situations and many areas, we face the double problem of estimating the risk of lying in some marked zone and, at the same time, the cost associated with it. To fix ideas, we may be interessed in estimating the immunocompromised patients number $Q$, and the size of the set $\mathcal{M}$ of infected people, in some population $\mathcal{P}$. At the same time, we know that the severity of the infection is measured by the viral load $Y$ expressed in RNA copies per milliliter of blood plasma. The cost of treatement, for example a course of chemotherapy, heavily depends on the viral load. If one has to treat all the patients, there is a cost to pay for each treatment, 
which is a cost function $d(Y)$. Facing these two problems at the same time, comparing two different populations or monitoring the evolution of the global situation should be based on the couple $(Q, d(Y))$ rather than on which is commonly called the HIV/AIDS adult prevalence rate, on what is based international comparison. In order to make a workable statistic, consider a sample of individuals $\mathcal{E}=\{1,2, \cdots, n\}$ drawn for $\mathcal{P}$ and measure the viral load $Y_{j}$ for each $j \in \mathcal{M}$. A general comparative statistic should be of the form

$$
\sum_{j \in \mathcal{M}} d\left(Y_{j}\right)
$$

Since comparisons over the time are based on this index, one will be interested in putting more or less emphasis on the more infected or not, in terms of viral load. This is achieved by affecting a weight $\rho(j)$ to $j \in \mathcal{M}$ as a monotone function of the rank $R_{j, n}$ of $Y_{j}$ in the sample. For an increasing $\rho$, it is paid more attention to less infected while the contrary holds for a decreasing one. This leads to statistics like

$$
J_{n}(\mathcal{M}, d, \rho)=\frac{1}{n} \sum_{j \in \mathcal{M}} \rho\left(R_{j, n}\right) d\left(Y_{j}\right) .
$$

It is also known that the viral load is detectable only above a threshold of value $Z_{0}=40$ RNA copies per milliliter of blood plasma. We thus have

$$
j \in \mathcal{M} \Leftarrow Y_{j} \geq Z_{0}
$$

and

$$
J_{n}(\mathcal{M}, d, \rho)=\frac{1}{n_{Y_{j} \geq Z_{0}}} \rho\left(R_{j, n}\right) d\left(Y_{j}\right) .
$$

We may decide to concentrate on the very expansive chemotherapy courses due to financial pressure. In that case, we change the threshold to $Z>Z_{0}$ according to the available budget.

Such statistics are also used in insurance theory. Suppose that one insurance company receives $n$ claims $\left\{Y_{1}, \cdots, Y_{n}\right\}$. We may fix a threshold $Z$ such that any claim greater than $Z$ is seen as causing a loss $\left(Y_{j}-Z\right)$ for the company. It then becomes interesting to estimate the number of possible claims over $Z$,

$$
Q_{n}=\sum_{j=1}^{n} 1_{\left(Y_{j} \geq Z\right)}
$$

and to choose a distorsion function $\gamma$ of the individual loss $\left(Z-Y_{j}\right)$; hence, (1) is transformed here into

$$
J_{n}(Z, d, \rho)=\frac{1}{n} \sum_{Y_{j} \geq Z} \rho\left(R_{j, n}\right) \gamma\left(Y_{j}\right)=\frac{1}{n} \sum_{j \geq n-Q} \rho(j / n) d\left(Y_{j, n}-Z\right),
$$

where $Y_{1, n} \leq \cdots \leq Y_{n, n}$ are the order statistics based on $\left\{Y_{1}, \cdots, Y_{n}\right\}$. In this case, $J_{n}(\mathcal{M}, d, \rho)$ may be seen as a risk measure.

In poor countries, an individual is considered as a poor one when his income $Y$ below some threshold $Z$, called poverty line. And then

$$
Q_{n}=\sum_{j=1}^{n} 1_{\left(Y_{j} \leq Z\right)}
$$

is the total number of poor people in the sample, while $Q_{n}$ is the poor headcount. Usually the cost function here depends on the relative poverty gap $\gamma\left(Y_{j}\right)=\left(Z-Y_{j}\right) / Z$. In this field, following Lo [1], $J_{n}(\mathcal{M}, d, \rho)$ may be called a General Poverty Index (GPI). The same form may also be used in medical science when dealing with vitamine (say vitamine $\mathrm{D}$ ) deficiency. In this case, $J_{n}(Z, d, \rho)$ is used as a general measure of vitamine deficiency to evaluate the mean cost of vitamine supply as a treatment.

We see from the lines above that (1) is a very general statistic, which works in various fields, with losses or gains dependent on the meaning of the cost function $c$. We are entitled to name it as a Weighted Mean Loss or Gain (WMLG) statistic or random measure or index. It may take a specific name, depending on the particular field where it operates. In the loss (resp. gain) case, we simply denote it WML (resp. WMG).

When we have time-dependent data, over the time $[0, T]$ with continuous observations 
$\left(\left\{Y_{1}(t), \cdots, Y_{n}(t)\right\}, t \in T\right)$, we are led to a time-dependent WMLG statistic in the form

$$
J_{n}(\mathcal{M}, \gamma, \rho, t)=\frac{1}{n} \sum_{j \in \mathcal{M}} \rho\left(R_{j, n}(t)\right) d\left(Y_{j}(t)\right)
$$

In the case where $\mathcal{M}$ is based on the threshold $Z$; the latter should eventually depend on the time and becomes $Z=Z(t)$. Also, in an spatial analysis, it would be possible to have a particular threshold for any area. The choice of $d$ and $\rho$ depends of the specific role played by (1). But, a set of axioms, which are desirable or mandatory to be fulfilled for a welfare or a risk measure, is usually adopted. For risk measures, such axiomes alongside an axiomatic foundation are to be found in Artzner et al. [2]. For poverty analysis, a large and deep review of the axiomatic approach, due to Sen [3], is available in Zheng [4].

Finally, taking into account various forms of (1) in the literature, the following form of threshold-based weighted mean loss seems to be a general one

$$
J_{n}(Z, \omega, d)=\frac{A(n)}{n B_{n}\left(Q_{n}\right)} \sum_{j=1}^{Q_{n}} w\left(\mu_{1} n+\mu_{2} Q_{n}-\mu_{3} j+\mu_{4}\right) d\left(\left(Z-Y_{j, n}\right) / Z\right)
$$

or the following

$$
J_{n}^{*}(Z, \omega, d)=\frac{A(n)}{n B_{n}\left(Q_{n}\right)} \sum_{j \geq n-Q}^{n} w\left(\mu_{1} n+\mu_{2} Q_{n}-\mu_{3} j+\mu_{4}\right) d\left(\left(Z-Y_{j, n}\right) / Z\right),
$$

depending on whether we handle loss (with $Q_{n}$ defined in (3)) or gains (with $Q_{n}$ defined in (2)), and where

$$
B\left(Q_{n}\right)=\sum_{j=1}^{Q_{n}} w(j) .
$$

From a mathematical point of view, the asymptotic behaviors of the two forms radically differ although the writing seems symetrical. The reason is that for the first, the random variables used in (4) are bounded and the asymptotic handling is much easier. As for (5), we should face heavy tail problems and further complications may arise.

This paper is aimed at offering a full functional weak theory according to the most recent setting of such theories as stated in [5]. Particularly, we are interested here in the time-dependent investigation of (4), and next the functional weak theory in $d$ and $w$. We call the first class of statistics Upper Threshold Based Weighted Mean Loss or Gain (UTB WMLG) ones and the others are named Lower Threshold Based Weighted Mean Loss indices (LTB WMLG). This paper is only concerned with the first class of statistics. The others will be objects of further studies.

Consider for a while that $w$ and $d$ are fixed as well as the time. We notice that asymptotic results of $J_{n}(Z, \omega, d)$ are available for specific forms in Welfare theory or in Actuarial Sciences. For example, Lo [1] proved that

$$
\begin{aligned}
J_{n}(Z, \omega, d) \rightarrow J(Z, \omega, d) & =\int_{0}^{Z} w_{G}(Z, y) \mathrm{d}((z-y) / z) \mathrm{d} y \\
& =\mathbb{E}_{G}\left(w_{G}(Z, Y) d((Z-Y) / z) \mathbb{I}(Y \leq Z)\right) \\
& =\mathbb{E} w m g l,
\end{aligned}
$$

where $\mathbb{E} w m g l$ may be called the Exact UTB WMLG. For instance, the weight $w_{G}(Z, y)=2(1-y)$ is related to the Shorrocks [6] and Thon [7] statistics, $w_{G}(Z, y)=2(1-y / Z)^{k}$ is the Kakwani weight (see [8]), that includes the Sen [3] one corresponding to $k=1$. For $w_{G}(Z, y)=1$, we get the nonweighted mean losses or gains.

To be able to base statistical tests of such results, we may be interested in finding the asymptotic law of

$$
\sqrt{n}\left(J_{n}(\omega, d)-J(\omega, d)\right) \text { as } n \rightarrow \infty .
$$

However, we still need to handle longitidunal data, where the risk situation is analysed over a continuous period of time $[0, T]$. In this case, we are faced with continuous data in the form of $\{Y(t), 0<t<T\}$, and some modification is needed in the definition of indices to take this into account. We are then led to consider the 
time-dependent and UTB WMLG statistic defined by

$$
J_{n}(t)=\frac{A\left(Q_{n}(t), n, Z(t)\right)}{n B\left(Q_{n}(t)\right)} \sum_{j=1}^{Q_{n}(t)} w\left(\mu_{1} n+\mu_{2} Q_{n}(t)-\mu_{3} j+\mu_{4}\right) d\left(\frac{Z(t)-Y_{j, n}(t)}{Z(t)}\right),
$$

with $0 \leq t \leq T$ and $T \in \mathbb{R}$.

Instead of analysing such UTB WMLG for some specific functions $w$ or $d$, or at a fixed point $t$, it may be more valuable to have at once a uniform weak theory on $w, d$ and $t \in T$. Such a result will provide individual tests, and enables spatial and temporal comparisons of the risk measure. As well, since all the measure are expressed in the same Gaussian field, we have joint asymptotic distributions of the different indices themselves.

This paper is aimed at settling the uniform weak convergence of such statistics, which is the asymptotic theory of the time-dependent poverty measures $(6)$, in the space $C([0, T])$ of real continuous functions defined on $[0, T]$. First attempts were treated for the special case of time-dependent nonweighted mean loss or gain (MLG) measures in [9] and, in [10], for nonrandomly WMLG statistics, that is, WMLG statistics for which the weight is nonrandom, like the Shorrocks one, is dealt with. Now, we target to give here the most general results on the time-dependent UTB-WMLG statistics. Two potential applications areas here are vitamine deficiency risk measures and poverty measures. It is then natural to consider a threshold depending on the time. But we suppose that it lies in some finite interval

$$
0<Z_{1} \leq Z(t) \leq Z_{2}<+\infty .
$$

An important application is the statistical estimation of the Relative Mean Loss Variation (RMLV) from time $t$ to $s$ defined as follow

$$
\Delta R J(t, s)=(J(s)-J(t)) / J(t)
$$

by confidence intervals where $J_{n}(t)$ is a poverty measure, one of the Millennium Development Goals (MDG) is halving of extreme poverty from $t=2000$ to time $s=2015$. This means that we target to have $\Delta R J(t, s) \leq$ $-50 \%$. Our results below tackle this issue.

We will need a number of hypotheses towards an adequate frame for our study. These hypotheses may appear severe and numerous, at first sight, but most of them are natural and easy to get. We first need the following shape conditions for the WMLG measures themselves. The letter $S$ in the hypotheses names refers to shape conditions.

(HS1) There exist functions $h(p, q)$ of $(p, q) \in \mathbb{N}^{2}, c(u, v)$ and $\pi(u, v)$ of $(u, v) \in(0,1)^{2}$ independent of $t \in[0, T]$, such that, as $n \rightarrow+\infty$,

$$
\sup _{t \in[0, T]} \max _{1 \leq j \leq Q_{n}(t)}\left|A\left(n, Q_{n}(t)\right) h^{-1}\left(n, Q_{n}(t)\right) w\left(\mu_{1} n+\mu_{2} Q_{n}(t)-\mu_{3} j+\mu_{4}\right)-c\left(Q_{n}(t) / n, j / n\right)\right|=o_{P}^{*}\left(n^{-1 / 2}\right),
$$

where $o_{P}^{*}(1)$ denotes the convergence to zero in outer probability (see [5]).

(HS2)

$$
\sup _{t \in[0, T]} \max _{1 \leq j \leq Q_{n}(t)}\left|w(j) h^{-1}\left(n, Q_{n}(t)\right)-\frac{1}{n} \pi\left(Q_{n}(t) / n, j / n\right)\right|=o_{P}^{*}\left(n^{-3 / 2}\right)
$$

(HS3) There exists a function $c(u, v)$ of $(u, v) \in(0,1)^{2}$ independent of $t \in[0, T]$, such that, as $n \rightarrow+\infty$,

$$
\sup _{t \in[0, T]} \max _{1 \leq j \leq Q_{n}(t)}\left|A\left(n, Q_{n}(t)\right) B^{-1}\left(n, Q_{n}(t)\right) w\left(\mu_{1} n+\mu_{2} Q_{n}(t)-\mu_{3} j+\mu_{4}\right)-c\left(Q_{n}(t) / n, j / n\right)\right|=o_{P}^{*}\left(n^{-1 / 2}\right) .
$$

We will require other assumptions depending on the regularity of the functions $c$ and $\pi$. The letter $R$ in these hypotheses name refers to Regularity conditions..

(HR1) The bivariate functions $c$ and $\pi$ have equi-continuous partial differential on $(\beta, \xi) \times(0,1)$, where $\beta$ and $\xi$ are two real numbers to be defined later on.

(HR2) For a fixed $x$, the functions $y \rightarrow \frac{\partial c}{\partial y}(x, y)$ and $y \rightarrow \frac{\partial \pi}{\partial y}(x, y)$ are monotone.

(HR3) There exist $H_{0}>0$ and $H_{\infty}<+\infty$ such that, for $t \in[0, T]$,

$$
H_{0}<H_{c}(t)=\int c\left(G_{t}(Z(t)), G_{t}(y)\right) \gamma_{t}(y) \mathrm{d} G_{t}(y)<H_{\infty},
$$


and

$$
H_{0}<H_{\pi}(t)=\int \pi\left(G_{t}(Z(t)), G_{t}(y)\right) e_{t}(y) \mathrm{d} G_{t}(y)<H_{\infty},
$$

Our final achievement is that, when putting $J(t)=H_{c}(t) / H_{\pi}(t)$, we are able to get the uniform asymptotic law of $\left\{\sqrt{n}\left(J_{n}(t)-J(t)\right), 0 \leq t \leq T\right\}$ and to describe the limiting Gaussian process $\{\mathbb{G}(t), 0 \leq t \leq T\}$. This enables the statistical uniform estimates of $\Delta_{n} J_{n}(t, s)=J_{n}(t)-J_{n}(s)$ by $\Delta J(t, s)=J(t)-J(s)$ by interval confidences. We also particularize the results for the so-important Kakwani class of WMLG statistics of which the Sen one is a member. The results that have directly been derived for the Shorrocks case are rediscovered here.

\section{Our Results}

Our results will rely on the representation of Theorem [11], which in turn will need the following assumptions.

(HL1) There exist $\beta>0$ and $0<\xi<1$ such that

$$
0<\beta<\inf _{0 \leq t \leq T} G_{t}\left(Z_{1}\right)<\sup _{0 \leq t \leq T} G_{t}\left(Z_{2}\right)<\xi<1 .
$$

(HL2) The subclass $\mathcal{F}_{0}=\left\{\pi_{t, Z}: x \rightsquigarrow 1_{(x(t) \leq Z)}, t \in[0, T]\right\}$ of $\ell^{\infty}(C([0, T]))$, the set of real bounded and continuous functions, is a $\mathbb{P}_{Y}$-Glivenco-Cantelli class, that is, as $n \rightarrow \infty$,

$$
\sup _{t \in[0, T]}\left|G_{t, n}(Z(t))-G_{t}(Z(t))\right| \rightarrow 0 \text {, a.s.o.p. }
$$

where, for any $t \in[0, T]$ and $y \in \mathbb{R}, G_{t, n}(y)=n^{-1} \sum_{i=1}^{n} 1_{(Y(t) \leq y)}$. As a reminder $\mathbb{R} \ni z_{n} \rightarrow 0$, a.s.o.p as $n \rightarrow+\infty$ means $\left(z_{n} \rightarrow 0\right.$ in outer probability), that is : there exists a sequence of measurable random variables, $u_{n}$ such that for any $n \geq 1,\left|z_{n}\right| \leq u_{n}$ and $u_{n} \rightarrow 0$ as $n \rightarrow+\infty$.

Finally let us denote $f_{t}(x)=x(t)$, where $x \in \ell^{\infty}(C([0, T]))$.

(HL3) For any $t \in[0, T], G_{t}$ is strictly increasing and the functions $G_{t}$ are uniformly continuous in $t \in[0, T]$.

(HL4) $d$ is bounded by one and is differentiable with derivative function $d^{\prime}$ bounded by $\mathrm{M}: 0 \leq d \leq 1$, $\left|d^{\prime}\right| \leq M$.

Theorem 1 Suppose that (HS1)-(HS2), (HR1)-(HR3) and (HL1)-(HL4) hold. Put $J(t)=H_{c}(t) / H_{\pi}(t)$,

$$
\begin{aligned}
& K_{c}(t)=\int_{0}^{1} \frac{\partial c}{\partial x}\left(G_{t}(Z(t)), s\right) \gamma_{t}\left(G_{t}^{-1}(s)\right) \mathrm{d} s, \\
& K_{\pi}(t)=\int_{0}^{1} \frac{\partial \pi}{\partial x}\left(\left(G_{t}(Z(t)), s\right) e_{t}\left(G_{t}^{-1}(s)\right) \mathrm{d} s .\right. \\
& K(t)=H_{\pi}^{-1}(t) K_{c}(t)-H_{c}(t) H_{\pi}^{-2}(t) K_{\pi}(t), \\
& g_{c, t}(\cdot)=c\left(G_{t}(Z(t)), G_{t}\left(f_{t}(\cdot)\right)\right) \gamma_{t}\left(f_{t}(\cdot)\right), \\
& g_{\pi, t}=\pi\left(G_{t}(Z(t)), G_{t}\left(f_{t}(\cdot)\right)\right) e_{t}\left(f_{t}(\cdot)\right)+K(t) e_{t}\left(f_{t}(\cdot)\right) .
\end{aligned}
$$

and

$$
\begin{aligned}
& v_{c, t}(y)=\frac{\partial c}{\partial y}\left(G_{t}(Z(t)), G_{t}\left(f_{t}(y)\right)\right) \gamma_{t}\left(f_{t}(y)\right), \\
& v_{\pi, t}(y)=\frac{\partial \pi}{\partial y}\left(G_{t}(Z(t)), G_{t}\left(f_{t}(y)\right)\right) e_{t}\left(f_{t}(y)\right) .
\end{aligned}
$$

Define

$$
g_{t}=H_{\pi}^{-1}(t) g_{c, t}-H_{c, t}(t) H_{\pi, t}^{-2} g_{\pi, t}
$$


and

$$
v_{t}=H_{\pi}^{-1}(t) v_{c, t}-H_{c}(t) H_{\pi}^{-2}(t) v_{\pi, t} .
$$

Then we have, uniformly in $t \in[0, T]$, the following representation, as $n \rightarrow \infty$,

$$
\sqrt{n}\left(J_{n}(t)-J(t)\right)=\alpha_{t, n}\left(g_{t}\right)+\beta_{n}\left(v_{t}, t\right)+o_{P}^{*}(1),
$$

with

$$
\alpha_{t, n}\left(g_{t}\right)=\frac{1}{\sqrt{n}} \sum_{j=1}^{n}\left\{g_{t}\left(Y_{j}(t)\right)-\mathbb{E} g_{t}\left(Y_{j}(t)\right)\right\} \text {. }
$$

and

$$
\beta_{n}\left(v_{t}, t\right)=\frac{1}{\sqrt{n}} \sum\left\{G_{t, n}\left(Y_{j}(t)\right)-G_{t}\left(Y_{j}(t)\right)\right\} v_{t}\left(Y_{j}(t)\right),
$$

Suppose that (HS3), (HR1)-(HR3) and (HL1)-(HL4) hold. Then, (10) holds with

$$
K(t)=K_{c}(t), \quad g_{t}=g_{c, t} \text { and } v_{t}=v_{c, t} .
$$

This theorem expresses our studied time-dependent statistics as the sum of a functional empirical process and the stochastic process (11). It will be seen, for a fixed time, that $\beta_{n}\left(v_{t}, t\right)$ is asymptotically an integral of the quantile process $\sqrt{n}\left(s-V_{t, n}(s)\right)$ based on $G_{t}\left(Y_{1}(t)\right), \cdots, G_{t}\left(Y_{n}(t)\right)$ (where $V_{t, n}(s)$ is the empirical quantile function) and then of empirical process $\alpha_{t, n}(s)=\sqrt{n} \sum\left(I_{G_{t}\left(Y_{j}(t) \leq s\right)}-s\right)$, These facts make easy the handling of $\sqrt{n}\left(J_{n}(t)-J(t)\right)$ in the modern empirical process setting as stated in [5]. We still need a thorough study of (11) and its connection with $\alpha_{t, n}$ while the computation of the variance and covariance function. This is done separately in [12] to avoid lengthy papers.

Now, we use these tools to give first, general laws for the WMLG statistic below and then for the Kakwani class of indices in Section 2.2 and for the Shorrocks-Thon indices in Section 3. We finish by a special study of the absolute and the relative poverty changes in Section 4.

While we deal with the general index and we use the outcomes of Theorem 1, we adopt the following writing:

$$
\alpha_{t, n}\left(g_{t}\right)=\frac{1}{\sqrt{n}} \sum_{j=1}^{n}\left(W_{j}(t)-\mathbb{E}_{j} W_{j}(t)\right)
$$

where $W_{j}(t)=g_{t}\left(Y_{j}(t)\right)$. Then we are entitled to express the hypotheses (HT1) and (HT2) below on the $W_{j}(t)$ in place of the $Y_{j}(t)$ for the general case. And we suppose that $W_{j}(t)$ admits a density probability $m_{t}$ for each $t \in[0, T]$. In particular cases, we will turn back to hypotheses on the $Y_{j}(t)$ for establishing (HT2) and (HT3) and subsequently recover the results. In the sequel, $r$ is a fixed positive real number such that $0<r<1 / 2$. And from now, the limits and the $o_{P}^{*}(1)$ are performed when $n \rightarrow \infty$.

(HT1) For $0 \leq s, t \leq T$, for some constant $K, \mathbb{E}|W(t)-W(s)|^{2} \leq K|t-s|^{1+r}$.

(HT2) For $0 \leq s, t \leq T$, for some constant $K,|\mathbb{E}(W(t))-\mathbb{E}(W(s))|^{2} \leq K|t-s|^{1+r}$.

In order to define our last assumption, we need the following functions:

$$
g\left(v_{t}, v_{s}, t, s\right)=\int\left(\int_{x \geq u} v_{t}(x) \mathrm{d} G_{t}(x)\right)\left(\int_{y \geq v} v_{s}(y) \mathrm{d} G_{s}(y)\right) \mathrm{d} G_{t, s}(u, v)
$$

and

$$
c\left(v_{t}, t\right)=\int\left(\int_{x \geq u} v_{t}(x) \mathrm{d} G_{t}(x)\right)^{2} \mathrm{~d} G(u) .
$$

with, by convention, $\mathbb{E}_{t} h=\int h(u) \mathrm{d} G_{t}(u)$ for a function $h$. Set

(HT3) If there is a universal constant $K_{3}$, such that for any $\delta>0$, for large enough values of $n$,

$$
|s-t| \leq \delta \Rightarrow\left|2\left(c\left(v_{t}, t\right)-g\left(v_{t}, v_{s}, t, s\right)\right)+\left\{\left(\mathbb{E}_{t} G_{t} v_{t}\right)\left(\mathbb{E}_{s} G_{s} v_{s}\right)-\left(\mathbb{E}_{t} G_{t} v_{t}\right)^{2}\right\}\right| \leq \frac{3}{2} K_{3}|s-t|^{1+r}
$$


We are now able to give our general main result.

Theorem 2 Assume the conditions of Theorem 1 hold and that (HT1)-(HT3) are satisfied. Then the stochastic process $\left\{\sqrt{n}\left(J_{n}(t)-J(t)\right), 0 \leq t \leq T\right\}$ converges in $\ell^{\infty}([0, T])$ to a centered Gaussian process $\{\mathbb{G}(t), 0 \leq t \leq T\}$ with covariance function

$$
\Gamma(t, s)=\Gamma_{1}\left(g_{t}, g_{s}, t, s\right)+\Gamma_{2}\left(v_{t}, v_{s}, t, s\right)+\Gamma_{2}(t, s)+\Gamma_{3}\left(g_{t}, v_{t}, t, s\right),
$$

with

$$
\begin{aligned}
& \Gamma_{1}\left(g_{t}, g_{s}, t, s\right)=\int\left(g_{t}(x)-\eta(t)\right)\left(g_{s}(y)-\eta(s)\right) \mathrm{d} G_{t, s}(x, y), \\
& \Gamma_{2}\left(v_{t}, v_{s}, t, s\right)=g\left(v_{t}, v_{s}, t, s\right)-\left(\mathbb{E}_{t} G_{t} v_{t}\right)\left(\mathbb{E}_{s} G_{s} v_{s}\right), \\
& g\left(v_{t}, v_{s}, t, s\right)=\int\left(\int_{x \geq u} v_{t}(x) \mathrm{d} G_{t}(x)\right)\left(\int_{x \geq v} v_{s}(x) \mathrm{d} G_{s}(x)\right) \mathrm{d} G_{t, s}(u, v),
\end{aligned}
$$

and

$$
\Gamma_{3}\left(g_{t}, v_{s}, t, s\right)=\kappa\left(g_{t}, v_{s}, t, s\right)+\kappa\left(g_{s}, v_{t}, s, t\right),
$$

with

$$
\kappa\left(g_{t}, v_{s}, t, s\right)=\int g_{t}(u)\left(\int_{x \geq v} v_{s}(x) \mathrm{d} G_{s}(x)\right) \mathrm{d} G_{t, s}(u, v),
$$

and $g_{t}$ and $v_{t}$ are given in Theorem 1, and

$$
\eta(t)=\int g_{t}(y) \mathrm{d} G_{t}(y) .
$$

Proof. We have to do three things. First, we show that $\sqrt{n}\left(J_{n}(t)-J(t)\right)$ is asymptotically tight. Next, we have to prove that it converges in finite distributions. And finally, we should compute the covariance function. We will only sketch the first and the second tasks with the appropriate citations. The second will be properly adressed.

Since the assumptions of Theorem 1 hold, we have the representation (10). Put

$$
N_{n}(t)=\alpha_{t, n}\left(g_{t}\right)+\beta_{n}\left(v_{t}, t\right),
$$

First (HT2) and (HT2) yield, for each $j \in[1, n]$, for some constant $K$,

$$
\mathbb{E}\left|W_{j}(t)-W_{j}(s)\right|^{2}+\left|\mathbb{E} W_{j}(t)-\mathbb{E} W_{j}(s)\right|^{2} \leq K|s-t|^{1+r},
$$

and hence, by repeated use of $c_{2}$-inequality (that is, for any couple $(a, b)$ of scalars $|a+b|^{2} \leq 2\left(|a|^{2}+|b|^{2}\right)$, for some constant $K$,

$$
\left|\alpha_{t, n}\left(g_{t}\right)-\alpha_{s, n}\left(g_{s}\right)\right|^{2} \leq K|s-t|^{1+r}
$$

We remind again that $r$ is strictly less that $1 / 2$, otherwise functions satisfying 15 are constant. Here and in the sequel, $K$ is a generic constant eventually taking different values from one formula to another. Next, we find in [12], that $\mathbb{E}\left(\beta_{n}\left(v_{t}, t\right)-\beta_{n}\left(v_{s}, s\right)\right)^{2}$ is

$$
2\left(c\left(v_{t}, t\right)-g\left(v_{t}, v_{s}, t, s\right)\right)+\left\{\left(\mathbb{E}_{t} G_{t} v_{t}\right)\left(\mathbb{E}_{s} G_{s} v_{s}\right)-\left(\mathbb{E}_{t} G_{t} v_{t}\right)^{2}\right\}+\frac{K_{n}(t, s)}{n},
$$

where $K_{n}(t, s)$ is bounded uniformly in $n, t$ and $s$. So by combining (14), (15), (16) and (HT3), and by the $c_{2}$-inequality, we get for some $K$ that for any $\delta>0$, for large enough values of $n$,

$$
|s-t| \leq \delta \Rightarrow\left|N_{n}(t)-N_{n}(s)\right|^{2} \leq K_{4}|s-t|^{1+r},
$$

Thus $\left\{N_{n}(t), t \in[0, T]\right\}$ is asymptotically tight by Lemma 1 in [9], which is an adaptation of Example 2.2.12 in [5]. To finish the proof, we have to establish that finite-distributions of $N_{n}(t)$ converge to those of some Gaussian tight process $\mathbb{G}$. For simplicity's sake, we do it in the two dimensional case, for 
$\left(N_{n}\left(t_{1}\right), N_{n}\left(t_{2}\right)\right)$. Consider $N=a N_{n}\left(t_{1}\right)+b N_{n}\left(t_{2}\right)$. Still for simpicity's sake, let us set

$$
N_{n}\left(t_{1}\right)=\frac{1}{\sqrt{n}} \sum_{j=1}^{n} g_{1}\left(X_{j}\right)+\frac{1}{\sqrt{n}} \sum_{j=1}^{n}\left(G_{1, n}\left(X_{j}\right)-G_{1}\left(X_{j}\right)\right) v_{1}\left(X_{j}\right) \text {, }
$$

and

$$
N_{n}\left(t_{2}\right)=\frac{1}{\sqrt{n}} \sum_{j=1}^{n} g_{2}\left(Y_{j}\right)+\frac{1}{\sqrt{n}} \sum_{j=1}^{n}\left(G_{2, n}\left(Y_{j}\right)-G_{2}\left(Y_{j}\right)\right) v_{2}\left(Y_{j}\right)
$$

where the $\left(X_{j}, Y_{j}\right)^{\prime} s$ stand for the $\left(Y_{j}\left(t_{1}\right), Y_{j}\left(t_{2}\right)\right)^{\prime} s$ as independent observations of $(X, Y), G_{1, n}$ (resp. $G_{2, n}$ ) is the empirical function based on $X_{1}, \cdots, X_{n}$ (resp. $Y_{1}, \cdots, Y_{n}$ ). Put

$$
G(x, y)=P(X \leq x, Y \leq y), \quad G_{1}(x)=G(x,+\infty), \quad G_{2}(y)=G(+\infty, y) .
$$

Now let, for each $n \geq 1, \varepsilon_{1, n}$ (resp. $\varepsilon_{2, n}$ ) be the quantile processes based respectively on $G_{1}\left(X_{1}\right), G_{1}\left(X_{2}\right), \cdots, G_{1}\left(X_{n}\right)$ (resp. $G_{2}\left(Y_{1}\right), G_{2}\left(Y_{2}\right), \cdots, G_{2}\left(Y_{n}\right)$ ). It is not hard to see that

$$
n^{-1 / 2} \sum_{j=1}^{n}\left(G_{1, n}\left(X_{j}\right)-G_{1}\left(X_{j}\right)\right) v_{1}\left(X_{j}\right)=\int_{0}^{1}-\varepsilon_{1, n}(s) v_{1}\left(G_{1}^{-1}(s)\right) \mathrm{d} s+o_{P}^{*}(1)
$$

and

$$
n^{-1 / 2} \sum_{j=1}^{n}\left(G_{2, n}\left(Y_{j}\right)-G_{2}\left(Y_{j}\right)\right) v_{2}\left(Y_{j}\right)=\int_{0}^{1}-\varepsilon_{2, n}(s) v_{2}\left[G_{2}^{-1}(s)\right] \mathrm{d} s+o_{P}^{*}(1) .
$$

Now, let $\alpha_{1, n}$ and $\alpha_{2, n}$ be the empirical processes based respectively on $G_{1}\left(X_{1}\right), G_{1}\left(X_{2}\right), \cdots, G_{1}\left(X_{n}\right)$ and on $G_{2}\left(Y_{1}\right), G_{2}\left(Y_{2}\right), \cdots, G_{2}\left(Y_{n}\right)$. We have (see [13], p. 584) that $\alpha_{i, n}(s)=-\varepsilon_{i, n}(s)+o_{P}^{*}(1)$ uniformly in $s \in(0,1)$, which gives

$$
\varepsilon_{n}(s, t)=\left(\varepsilon_{1, n}(s), \varepsilon_{2, n}(t)\right)=-\left(\alpha_{1, n}(s), \alpha_{2, n}(t)\right)+o_{P}^{*}(1),
$$

uniformly in $(s, t) \in(0,1)^{2}$. Now let us consider the functional empirical process $\alpha_{n}$ based on the $N_{j}=\left(G_{1}\left(X_{j}\right), G_{2}\left(Y_{j}\right)\right)^{\prime} s$, that is

$$
\alpha_{n}(F)=\frac{1}{\sqrt{n}} \sum_{j=1}^{n} f\left(N_{j}\right)-\mathbb{E} f\left(N_{j}\right)
$$

where $f$ a real function defined on $(0,1)^{2}$ such that $\mathbb{E} f\left(N_{i}\right)^{2}<\infty$. Finally, let $\gamma_{n}$ the fonctional empirical process based on the $\left(X_{i}, Y_{i}\right)^{\prime} s$, defined for $h: \mathbb{R}^{2} \rightarrow \mathbb{R}$,

$$
\gamma_{n}(H)=\frac{1}{\sqrt{n}} \sum_{j=1}^{n}\left\{h\left(X_{j}, Y_{j}\right)-\mathbb{E} h\left(X_{j}, Y_{j}\right)\right\}
$$

We have

$$
\alpha_{n}(f)=\gamma_{n}\left(h_{f}\right),
$$

for $h_{f}(x, y)=f\left(G_{1}(x), G_{2}(y)\right)$, We have by the classical results of empirical process that $\left\{\gamma_{n}(h), h \in \mathcal{H}\right\}$ converges to a Gaussian process $\{G(h), h \in \mathcal{H}\}$ whenever $\mathcal{H}$ is a Donsker class. It follows that $\left\{\gamma_{n}\left(1_{C}\right), C \in \mathcal{C}\right\}$ converges to a Gaussian process $\left\{G\left(1_{C}\right), C \in \mathcal{C}\right\}$ whenever $\mathcal{C}$ is a Vapnik-Cervonenkis class. But $\mathcal{C}=\left\{1_{]-\infty, x] \times]-\infty, y]},(x, y) \in \mathbb{R}^{2}\right\}$ is VC-class of index not greater than 2. (see [5] for VC-classes use to empirical processes). Thus putting $h_{x, y}=1_{]-\infty, x] \times]-\infty, y]}$, we have

$$
\gamma_{n}(x, y)=\gamma_{n}\left(h_{x, y}\right) \rightsquigarrow G\left(h_{x, y}\right)=\mathbb{G}(x, y),
$$

in $\ell^{\infty}\left(\mathbb{R}^{2}\right)$ where $\mathbb{G}$ is a tight Gaussian process such that

$$
\mathbb{E} \mathbb{G}\left(h_{1}\right) \mathbb{G}\left(h_{2}\right)=\int\left(h_{1}(x, y)-\mathbb{E}_{h_{1}}\right)\left(h_{2}(x, y)-\mathbb{E}_{h_{2}}\right) \mathrm{d} G(x, y) .
$$


Further, for $f_{1, s}=1_{[0, s] \times[0,1]}, \quad h_{f_{1, s}}(x, y)=1_{[0, s] \times[0,1]}\left(G_{1}(x), G_{2}(y)\right)=1_{\left.]-\infty, G_{1}^{-1}(s)\right] \times \mathbb{R}}(x, y)=h_{G_{1}^{-1}(s),+\infty}(x, y)$,

$$
\alpha_{1, n}(s)=\alpha\left(f_{1, s}\right)=\gamma_{n}\left(h_{G_{1}^{-1}(s),+\infty}\right),
$$

and for $h_{f_{2, t}}(x, y)=h_{+\infty, G_{2}^{-1}(t)}(x, y)$,

$$
\alpha_{2, n}(t)=\gamma_{n}\left(h_{+\infty, G_{2}^{-1}(t)}(x, y)\right)
$$

Now, by using the Skorohod-Wichura-Dudley Theorem, we are entitled to suppose that we are on a probability space such that

$$
\sup _{(x, y) \in R^{2}}\left|\gamma_{n}\left(h_{x, y}\right)-\mathbb{E}\left(h_{x, y}\right)\right| \rightarrow_{P} 0 .
$$

Now, since the functions $v_{i}$ are bounded, and putting $h_{1}(x, y)=g_{1}(x)$ and $h_{2}(x, y)=g_{2}(y)$, $N=a N_{n}\left(t_{1}\right)+n N_{n}\left(t_{2}\right)$ is equal to

$$
\left\{a \mathbb{G}\left(h_{1}\right)+b \mathbb{G}\left(h_{2}\right)\right\}+\int_{0}^{1}\left\{a \mathbb{G}\left(h_{G_{1}^{-1}(s),+\infty}\right) v_{1}\left(G_{1}^{-1}(s)\right)+b \mathbb{G}\left(h_{+\infty, G_{2}^{-1}(s)}\right) v_{2}\left(G_{2}^{-1}(s)\right)\right\} \mathrm{d} s+o_{P}^{*}(1) .
$$

One easily proves that

$$
\left\{a \mathbb{G}\left(h_{1}\right)+b \mathbb{G}\left(h_{2}\right)\right\}+\int_{0}^{1}\left\{a \mathbb{G}\left(h_{G_{1}^{-1}(s),+\infty}\right) v_{1}\left(G_{1}^{-1}(s)\right)+b \mathbb{G}\left(h_{+\infty, G_{2}^{-1}(s)}\right) v_{2}\left(G_{2}^{-1}(s)\right)\right\} \mathrm{d} s .
$$

is a Gaussian random variable since the second term is a Riemann integral, which is a limit of finite linear combinations of Gaussian random variables. Thus $N$ is asymptotically Gaussian. We are able to do the same for an arbitrary finite-distribution $\left(N_{n}\left(t_{1}\right), \cdots, N_{n}\left(t_{k}\right)\right)$, The computation of the limiting Gaussian process requires heavy calculations done in [12]. The proof ends with providing the covariance function $\Gamma_{1}$ of $\alpha_{t, n}\left(g_{t}\right), \Gamma_{1}$ of $\beta_{n}\left(v_{t}, t\right)$ and the covariance $\Gamma_{3}$ function between them.

\subsection{Special Cases}

Since the results are stated in a more general form and may appear very sophisticated, it seems necessary to show how they work for common cases. We apply our results to two key examples in Welfare analysis: the class of Kakwani's and Shorrocks' statistics. These two examples are particularly interesting since they put the emphasis on the less deprived individuals within the whole population (with weight $n-j+1$ ) for Shorrock's statistic), or within the marked individuals (with weight $Q-j+1$ ) for Kakwani's class of statistic including sen's measure). In both case, taking the weight at the power $k$ may lead to more accuracy in the statistical estimation.

\subsection{The Kakwani Case}

We are now applying the general results to the Kakwani WMLG statistics of parameter $k \geq 1$, defined by

$$
J_{k, n}(t)=\frac{Q_{n}(t)}{n \sum_{j=1}^{Q_{n}(t)} j^{k}} \sum_{j=1}^{Q_{n}(t)}\left(Q_{n}(t)-j+1\right)^{k} d\left(\frac{Z(t)-Y_{j, n}(t)}{Z(t)}\right) .
$$

The way we are using here is to be repeated for any particular index. For instance, the results in [9] and [10] may be rediscovered in this way. In this specific case, we turn the hypotheses (HT1) and (HT2) on $W_{j}^{\prime} s$ to the $Y_{j}^{\prime} s$ as follows. Suppose the d.f. $G_{t}(x)=\mathbb{P}\left(Y_{j}(t) \leq x\right)$ admits a derivative $m_{t}(x)$. Put $G_{s, t}(u, v)=\mathbb{P}(X(t) \leq u, X(s) \leq v)$. Introduce:

(H0) For $0 \leq s, t \leq T$, for some constant $K,|Z(s)-Z(t)|^{2} \leq K|t-s|^{1+r}$.

(H1) There exists a positive function $m$ such that for $0 \leq s, t \leq T, u \in \mathbb{R}, 0<r<1$ )

$$
\left|m_{t}(u)-m_{s}(u)\right| \leq m(u)|t-s|^{(1+r) / 2} \text { and } \int_{0}^{Z_{2}} m(u) \mathrm{d} u=K_{1}<\infty .
$$


and

$$
\sup _{x \in\left(Z_{1}, Z_{2}\right)}|m(x)|=M_{0}<\infty .
$$

(H2) For $0 \leq s, t \leq T$, for some constant $K$,

$$
\sup _{u \geq 0}\left|G_{t, s}(u, u)-G_{s}(u)\right| \leq K|t-s|^{1+r},
$$

and

$$
\left|G_{t}(Z(t))-G_{t}(Z(t) \wedge Z(s))\right| \leq K|t-s|^{1+r} .
$$

(H3) For $0 \leq s, t \leq T$, for some constant $K, \mathbb{E}|Y(t)-Y(s)|^{2} \leq K|t-s|^{1+r}$,

We check, in the Kakwani case, that the representation of Theorem 1 holds with $h\left(Q_{n}(t), n\right)=n^{k}$, $c(x, y)=(x-y)^{k}, \pi(x, y)=\frac{y^{k}}{x}$ and then

$$
\begin{gathered}
H_{\pi}(t)=G_{t}(Z(t))^{k} /(k+1), \\
H_{c}(t)=\int_{0}^{Z(t)}\left(G_{t}(Z(t))-G_{t}(y)\right)^{k} \gamma_{t}(y) \mathrm{d} G_{t}(y),
\end{gathered}
$$

so that

$$
J_{k}(t)=H_{c}(t) / H_{\pi}(t)=(k+1) \int_{0}^{Z(t)}\left(1-G_{t}(y) / G_{t}(Z(t))\right)^{k-1} \gamma_{t}(y) \mathrm{d} G_{t}(y) .
$$

Next

$$
K_{c}(t)=k \int_{0}^{Z(t)}\left(G_{t}(Z(t))-G_{t}(y)\right)^{k-1} \gamma_{t}(y) \mathrm{d} G_{t}(y), \quad K_{\pi}(t)=-G_{t}(Z(t))^{k-1} /(k+1),
$$

and then

$$
K(t)=(k+1) k\left\{G_{t}(Z(t))^{-k-1} r_{k-1}(t)+r_{k}(t)\right\},
$$

where

$$
r_{k}(t)=\int_{0}^{Z(t)}\left(G_{t}(Z(t))-G_{t}(y)\right)^{k} \gamma_{t}(y) \mathrm{d} G_{t}(y)
$$

For

$$
g_{t}(\cdot)=(k+1)\left(1-G_{t}\left(f_{t}(\cdot)\right) / G_{t}(Z(t))\right)^{k} \gamma_{t}\left(f_{t}(\cdot)\right)+K(t) 1_{\left(f_{t}(\cdot) \leq Z(t)\right)},
$$

and

$$
v_{t}(y)=-k(k+1) G_{t}(Z(t))^{-2 k-1}\left\{G_{t}(Z(t))^{k+1}\left(G_{t}(Z(t))-G_{t}(y)\right)^{k-1} \frac{Z(t)-y}{Z(t)}-(k+1) r_{k}(t) G_{t}(y)^{k-1}\right\} 1_{\left(f_{t}(\cdot) \leq Z(t)\right)} .
$$

we will get the representation

$$
\sqrt{n}\left(J_{k, n}(t)-J_{k}(t)\right)=N_{n}(t)+o_{P}^{*}(1)
$$

with

$$
N_{n}(t)=\alpha_{t, n}\left(g_{t}\right)+\beta_{n}\left(v_{t}, t\right)
$$

Theorem 3 Let (HL1), (HL3), (HL4), (H0)-(H3) hold. Then $\left\{\sqrt{n}\left(J_{n}(t)-J(t)\right), 0 \leq t \leq T\right\}$ converges in $\ell^{\infty}([0, T])$ to a centered Gaussian process with covariance function $\Gamma$ given in Theorem 2 .

Proof. We begin to remark that (H3) ensures that $\left\{\sqrt{n}\left(G_{t, n}(Z(t))-G_{t}(Z(t))\right), 0 \leq t \leq T\right\}$ is asymptocially tight and hence (HL2). It is then enough to show that (HT1) and (HT2) hold from (H0), (H1), (H2) and (H3). 
But this follows from routine calculations that we only sketch here. We place these calculation in the appendix.

\section{The Shorrocks-Thon-Like Case}

We apply our results to the Shorrocks-Thon WMLG statistics measures defined by

$$
J_{n}(t)=\frac{1}{n^{2}} \sum_{j=1}^{Q_{n}(t)}(2 n-2-j+1) \mathrm{d}\left(\frac{Z(t)-Y_{j, n}(t)}{Z(t)}\right)
$$

This is the Thon index. One obtains the Shorrocks one by replacing $1 / n^{2}$ by $1 /(n(n+1))$ We also check here that representation of Theorem 1 holds in the simple case corresponding to (HS3), with $c(x, y)=(x-y)$, In this case, $\pi$ is useless. Then

$$
\begin{gathered}
J(t)=H_{c}(t)=2 \int_{0}^{Z(t)}\left(1-G_{t}(y)\right) \gamma_{t}(y) \mathrm{d} G_{t}(y), \\
K(t)=K_{c}(t)=0, \quad v(y)=v_{c}(y)=-2 \gamma_{t}(y)
\end{gathered}
$$

Here again $\left\{\sqrt{n}\left(J_{n}(t)-J(t)\right), 0 \leq t \leq T\right\}$ has the same asymptotic behaviour described in Theorem 3 with

$$
g_{t}(y)=2\left(1-G_{t}(y)\right) \text { and } v(y)=v_{c}(y)=-2 \gamma_{t}(y)
$$

under the same hypotheses (HL1)-(HL4) and (H0)-(H3)

\section{Estimation of the WLMG Statistic Variation}

Although they are very expensive to collect, longitudinal data are highly preferred for adequate estimate of the absolute index variation $\Delta J(t, s)=J(s)-J(t)$, which is the exact measure of WMLG change between the periods $t$ and $s$ and the associate relative WMLG variation $\Delta R J(t, s)=(J(s)-J(t)) / J(t)$. Their respective natural estimators are of course $\Delta J_{n}(t, s)=J_{n}(s)-J_{n}(t)$ and $\Delta R J_{n}(t, s)=\left(J_{n}(s)-J_{n}(t)\right) / J_{n}(t)$ Our previous results yield the follow

Theorem 4 Under the assumptioms of Theorem 1 or Theorem 2,

$$
\sqrt{n}\left(\Delta J_{n}(t, s)-\Delta J(t, s)\right) \rightarrow \mathcal{N}\left(0, \Gamma_{4}(s, t)\right),
$$

where $\Gamma_{4}(s, t)=\Gamma(t, t)+\Gamma(s, s)-2 \Gamma(t, s)$, and

$$
\sqrt{n}\left(\Delta R J_{n}(t, s)-\Delta R J(t, s)\right) \rightarrow \mathcal{N}\left(0, \Gamma_{5}(t, s)\right)
$$

where

$$
\Gamma_{5}(t, s)=a_{1}^{2} \Gamma(t, t)+a_{2}^{2} \Gamma(s, s)+2 a_{1} a_{2} \Gamma(s, t)
$$

with

$$
\begin{aligned}
& a_{1}=-(1+\Delta R J(t, s)) / J(t), \\
& a_{2}=1 / J(t)
\end{aligned}
$$

The proof is straightforward. We also might consider the convergence of $\sqrt{n}\left(\Delta J_{n}(t, s)-\Delta J(t, s)\right)$ to the Gaussian process $\Delta \mathbb{G}(t, s)=\mathbb{G}(s)-\mathbb{G}(t)$ in $\ell^{\infty}\left([0, T]^{2}\right)$. Anyway for fixed $t$ and $s$,

$\sqrt{n}\left(\Delta J_{n}(t, s)-\Delta J(t, s)\right)$ converges to the Gaussian random variable $\Delta \mathbb{G}(t, s)=\mathbb{G}(t)-\mathbb{G}(s)$ by the continuity Theorem with $\Gamma_{4}(s, t)$ as variance. Also, by using the Skorohod-Wichura-Dudley Theorem, we have

$$
\sqrt{n}\left(\Delta R J_{n}(t, s)-\Delta R J(t, s)\right)=a_{2} \mathbb{G}(s)+a_{1} \mathbb{G}(t)+o_{P}^{*}(1)
$$

An important application of the second part of this theorem is related to checking the achievement of specific goals. One may, within a national or regional strategy, whish to have some deprivation limited to some extent. For example, the UN has assigned a number of goals, named Millennium Development Goals (MDG), to its members. We are concerned here by one of them. Indeed, it is whished to halve the extreme poverty in the world 
in year $s=2015$ starting from year $t=2000$. When the WMLG statistic is a poverty measure, we may use $\Delta R J(t, s)$ and check whether it is less than -0.5 . And an $(1-\alpha)$-confidence interval $I R(\alpha)$ based on these results is

$$
\left[\Delta R J_{n}(t, s)-n^{-1 / 2} \sqrt{\Gamma_{5}(s, t)} u_{1-\alpha / 2}, \Delta R J_{n}(t, s)+n^{-1 / 2} \sqrt{\Gamma_{5}(s, t)} u_{1-\alpha / 2}\right] \equiv\left[J^{0}(\alpha), J^{1}(\alpha)\right]
$$

where $\mathbb{P}\left(\mathcal{N}(0,1) \leq u_{1-\alpha / 2}\right)=\alpha$. This MDG will be reported achieved at the $95 \%$ level if the number $J^{1}(\alpha) \leq-0.5$.

\subsection{Datadriven Applications and Variance Computations}

We apply our results in Economics and Welfare analysis. Especially, we consider the household surveys in Senegal in 2011 (ESAM II) and in 2006 (EPS) from which we construct pseudo-panel data and apply our results.

\subsubsection{Variance Computations for Senegalese Data}

We apply our results to Senegalese data. We do not really have longitudinal data. So we have constructed pseudo-panel data of size $n=116$, from two surveys: ESAM II conducted from 2001 to 2002 and EPS from 2005 to 2006 . We get two series $X^{1}$ and $X^{2}$. We present below the values of $\Gamma_{I}(1,2)$ denoted here $\gamma(1)$, $\Gamma_{J}(1,2)$ denoted here $\gamma(2)$ and $\Gamma(1,2)$ denoted here $\gamma(3)$.

When constructing pseudo-panel data, we get small sizes like $n=116$ here. We use these sizes to compute the asymptotic variances in our results with nonparametric methods. In real contexts, we should use high sizes comparable to those of the real databases, that is around ten thousands, like in the Senegalese case. Nevertheless, we back on medium sizes, for instance $n=696$, which give very accurate confidence intervals as shown in Table 1.

Before we present the outcomes, let us say some words on the packages. We provide different $\mathrm{R}$ script files at http://www.ufrsat.org/lerstad/resources/sallmergslo01.zip.

The user should already have his data in two files data1.txt and data2.txt. The first script file named after gamma_mergslo1.dat provides the values of $\gamma(1), \gamma(2)$ and $\gamma(3)$ for the FGT measure for $\alpha=0,1,2$ and for the six inequality measures used here. The second script file named gamma_mergslo2.dat performs the same for the Shorrocks measure. Finally, gamma_mergslo3.dat concerns the kakwani measures. Unless the user uploads new data1.txt and data2.txt files, the outcomes should the same as those presented in Appendix.

\subsubsection{Analysis}

First of all, we find that, at an asymptotical level, all our inequality measures and poverty indices used here have decreased. When inspecting the asymptotic variance, we see that for the poverty index, the FGT and the Kakwani classes respectively for $\alpha=1, \alpha=2$ and $k=1$ and $k=2$ have the minimum variance, specially for $\alpha=2$ and $k=2$. This advocates for the use of the Kakwani and the FGT measures for poverty reduction evaluation.

\section{Table 1. Variations of the poverty indices.}

\begin{tabular}{cccc}
\hline Index J & $\Delta J(1,2)$ & $\Gamma_{4}(1,2)$ & $C I_{95 \%}(\Delta J(1,2))$ \\
\hline SHOR & -0.03024621 & 0.02353406 & {$[-0.04264967,-0.01985518]$} \\
KAK (1) & -0.02108905 & 0.01097123 & {$[-0.02982085,-0.01425729]$} \\
KAK (2) & -0.02055594 & 0.01007820 & {$[-0.02961271,-0.01469601]$} \\
FGT (0) & -0.05977098 & 0.3170756 & {$[-0.09355847,-0.009889805]$} \\
FGT (1) & -0.01859332 & 0.00922992 & {$[-0.02620413,-0.01192899]$} \\
FGT (2) & -0.00432289 & 0.0008381113 & {$[-0.007194404,-0.002892781]$} \\
\hline
\end{tabular}




\section{Conclusion}

We obtained asymptotic laws of the UTB WMLG statistics with in mind, among other targets, the uniform estimation of the variation $\Delta J(t, s)$ and the relative variation $\Delta R J(t, s)$. The results are only illustrated with simple datadriven applications to income databases in Senegal. This opens large datadriven application in whole economic areas. In the theoritical hand, the Lower Threshold Based weighted mean loss or gain statistics is to be studied in accordance with heavy tail conditions and to be applied in Insurance and HIV/VIH fields.

\section{References}

[1] Lo, G.S. (2013) The Generalized Poverty Index. Far East Journal of Theoretical Statistics, 42, 1-22. http://pphmj.com/journals/fjst.htm

[2] Artzner, P., Delbaen, F., Eber, J.M. and Heath, D. (1999) Coherent Measures of Risk. Mathematical Finance, 9, 203228. http://dx.doi.org/10.1111/1467-9965.00068

[3] Sen, A.K. (1976) Poverty: An Ordinal Approach to Measurement. Econometrica, 44, 219-231. http://dx.doi.org/10.2307/1912718

[4] Zheng, B. (1997) Aggregate Poverty Measures. Journal of Economic Surveys, 11, 123-162. http://dx.doi.org/10.1111/1467-6419.00028

[5] van der Vaart, A.W. and Wellner, J.A. (1996) Weak Convergence and Empirical Processes With Applications to Statistics. Springer, New York.

[6] Shorrocks, A.F. (1995) Revisiting the Sen Poverty Index. Econometrica, 63, 1225-1230. http://dx.doi.org/10.2307/2171728

[7] Thon, D. (1979) On Measuring Poverty. Review of Income and Wealth, 25, 429-440. http://dx.doi.org/10.1111/j.1475-4991.1979.tb00117.x

[8] Kakwani, N. (1980) On a Class of Poverty Measures. Econometrica, 48, 437-446. http://dx.doi.org/10.2307/1911106

[9] Sall, S.T. and Lo, G.S. (2009) Uniform Weak Convergence of the Time-Dependent Poverty Measure for Continuous Longitudinal Data. Brazilian Journal of Probability and Statistics, 24, 457-467. http://dx.doi.org/10.1214/08-BJPS101

[10] Lo, G.S. and Sall, S.T. (2009) Uniform Weak Convergence of Non Randomly Weighted Poverty Measures for Longitudinal Data. 57th ISI Session.

[11] Lo, G.S. and Sall, S.T. (2010) Asymptotic Representation Theorems for Poverty Indices. Afrika Statistika, 5, $238-244$.

[12] Lo, G.S. (2010) A Simple Note on Some Empirical Stochastic Process as a Tool in Uniform L-Statistics Weak Laws. Afrika Statistika, 5, 245-251.

[13] Shorack, G.R. and Wellner, J.A. (1986) Empirical Processes with Applications to Statistics. Wiley-Interscience, New York. 


\section{Appendix}

Put

$$
W(t)=g_{t}(Y(t))=W_{1}(t)+W_{2}(t)
$$

with

$$
W_{1}(t)=K(t) 1_{(Y(t) \leq Z(t))}
$$

and

$$
W_{2}(t)=(k+1)\left(1-G_{t}(Y(t)) / G_{t}(Z(t))\right)^{k} \gamma_{t}(Y(t))
$$

We have first to prove that for $i=1,2$,

$$
\mathbb{E}\left|W_{i}(s)-W_{i}(t)\right|^{2} \leq K|t-s|^{1+r}
$$

Based on the expression of $K(t)$ and on the facts that $r_{k}(t)$ and $G_{t}(u)$ for $Z_{1} \leq u \leq Z_{2}$ are uniformly bounded for $t \in[0, T]$, it suffices to prove that

$$
\left|G_{t}(Z(t))^{-k}-G_{s}(Z(s))^{-k}\right| \leq K|s-t|^{(1+r) / 2}
$$

for $k \geq 1$,

$$
\left|r_{k}(t)-r_{k}(s)\right| \leq K|s-t|^{(1+r) / 2}
$$

and

$$
\mathbb{E}\left|1_{(Y(t) \leq Z(t))}-1_{(Y(s) \leq Z(s))}\right|^{2} \leq K|t-s|^{1+r}
$$

This would help to conclude with the $c_{2}$-inequality that

$$
\mathbb{E}\left|W_{1}(s)-W_{1}(t)\right|^{2} \leq K|t-s|^{1+r}
$$

Let us establish (14). We have

$$
\begin{aligned}
& \left|G_{t}(Z(t))^{-k}-G_{s}(Z(s))^{-k}\right| \\
& \quad \leq\left|G_{t}(Z(t))^{-k}-G_{t}(Z(s))^{-k}\right|+\left|G_{t}(Z(s))^{-k}-G_{s}(Z(s))^{-k}\right| \\
& \quad \leq k|Z(t)-Z(s)| m_{t} Z(s, t) G_{t}(Z(s, t))^{-k-1}+k\left|G_{t}(Z(s))-G_{s}(Z(s))\right| B(s, t)^{-k-1},
\end{aligned}
$$

where $Z(s, t)$ lies between $Z(t)$ and $Z(s)$ and $B(s, t)$ lies between $G_{t}(Z(s))$ and $G_{s}(Z(s))$. We then get

$$
\begin{aligned}
\left|G_{t}(Z(t))^{-k}-G_{s}(Z(s))^{-k}\right| & \leq k \beta^{-2 k} \zeta^{k-1} K\left\{|Z(t)-Z(s)|+\left|G_{t}(Z(s))-G_{s}(Z(s))\right|\right\} \\
& \leq K|s-t|^{1+r / 2} .
\end{aligned}
$$

Now we show (15)

$$
\begin{aligned}
\left|r_{0}(t)-r_{0}(s)\right| & =\left|\int_{0}^{Z(t)} \gamma_{t}(u) m_{t}(u) \mathrm{d} u-\int_{0}^{Z(s)} \gamma_{s}(u) m_{s}(u) \mathrm{d} u\right| \\
& \leq \int_{0}^{Z(s) \wedge Z(t)}\left|\gamma_{t}(u) m_{t}(u)-\gamma_{s}(u) m_{s}(u)\right|+\int_{Z(s) \wedge Z(t)}^{Z(s)} \gamma_{t}(u) m_{t}(u) \mathrm{d} u+\int_{Z(s) \wedge Z(t)}^{Z(s)} \gamma_{s}(u) m_{s}(u) \mathrm{d} u .
\end{aligned}
$$

ince $\gamma_{s}$ is uniformly bounded, we have by (H0) and (H1),

$$
\left|\int_{Z(s) \wedge Z(t)}^{Z(t)} \gamma_{t}(u) m_{t}(u) \mathrm{d} u+\int_{Z(t) \wedge Z(s)}^{Z(s)} \gamma_{s}(u) m_{s}(u) \mathrm{d} u\right| \leq K|s-t|^{1+r / 2},
$$


Further

$$
\int_{0}^{Z(s) \wedge Z(t)}\left|\gamma_{t}(u) m_{t}(u)-\gamma_{s}(u) m_{s}(u)\right| \leq \int_{0}^{Z_{2}}\left|\gamma_{t}(u)-\gamma_{s}(u)\right| m_{t}(u) \mathrm{d} u+\int_{0}^{Z_{2}} \gamma_{s}(u)\left|m_{t}(u)-m_{s}(u)\right| \mathrm{d} u
$$

and, since $\gamma_{t}(x)=d((Z(t)-u) / Z(t))$, we get that

$$
\int_{0}^{Z(s) \wedge Z(t)}\left|\gamma_{t}(u)-\gamma_{s}(u)\right| m_{t}(u) \mathrm{d} u \leq \int_{0}^{Z(s) \wedge Z(t)}|Z(t)-Z(s)| B(s, t)^{-1} u \mathrm{~d}^{\prime}((Z(s, t)-u) / Z(s, t)) m_{t}(u) \mathrm{d} u,
$$

where $Z(s, t)$ lies between $Z(t)$ and $Z(s)$. Then

$$
\int_{0}^{Z(s) \wedge Z(t)}\left|\gamma_{t}(u)-\gamma_{s}(u)\right| m_{t}(u) \mathrm{d} u \leq K_{1} \beta^{-1} Z_{2}^{2}|Z(t)-Z(s)| \leq K|s-t|^{(1+r) / 2} .
$$

From (24)-(26), we conclude that

$$
\left|r_{0}(t)-r_{0}(s)\right| \leq K|s-t|^{(1+r) / 2}
$$

and for $k>1$,

$$
r_{k}(t)=\int_{0}^{Z(t) \wedge Z(s)}\left(G_{t}(y)-G_{t}(Z)\right)^{k} \gamma_{t}(u) m_{t}(u) \mathrm{d} u+\int_{Z(t) \wedge Z(s)}^{Z(t)}\left(G_{t}(y)-G_{t}(Z)\right)^{k} \gamma_{t}(u) m_{t}(u) \mathrm{d} u,
$$

and

$$
r_{k}(s)=\int_{0}^{Z(t) \wedge Z(s)}\left(G_{s}(y)-G_{s}(Z)\right)^{k} \gamma_{s}(u) m_{s}(u) \mathrm{d} u+\int_{Z(t) \wedge Z(s)}^{Z(s)}\left(G_{s}(y)-G_{s}(Z)\right)^{k} \gamma_{s}(u) m_{s}(u) \mathrm{d} u,
$$

with

$$
\left|\int_{Z(t) \wedge Z(s)}^{Z(t)}\left(G_{t}(y)-G_{t}(Z)\right)^{k} \gamma_{t}(u) m_{t}(u) \mathrm{d} u\right|
$$

and

$$
\left|\int_{Z(t) \wedge Z(s)}^{Z(s)}\left(G_{s}(y)-G_{s}(Z)\right)^{k} \gamma_{s}(u) m_{s}(u) \mathrm{d} u\right|
$$

less than $2 M_{0} \beta^{k}|Z(t)-Z(s)|$. Now $\left|r_{k}(t)-r_{k}(s)\right|$ is less than $A+B$ with

$$
A=\int_{0}^{Z(t) \wedge Z(s)}\left|\left(G_{t}(y)-G_{t}(Z)\right)^{k}-\left(G_{s}(y)-G_{s}(Z)\right)^{k}\right| \gamma_{s}(u) m_{s}(u) \mathrm{d} u,
$$

and

$$
B=\int_{0}^{Z(t) \wedge Z(s)}\left(G_{t}(y)-G_{t}(Z)\right)^{k}\left|\gamma_{s}(u) m_{s}(u)-\gamma_{t}(u) m_{T}(u)\right| \mathrm{d} u .
$$

By (H2), $A$ is less than $2 k Z_{2} \xi^{k-1}\left(\int_{0}^{Z_{2}} m(u) \mathrm{d} u\right)|s-t|^{(1+r) / 2}$ and $B \leq K|s-t|^{(1+r) / 2}$ by (24), (25) and (26). Then for $k \geq 1$,

$$
\left|r_{k}(t)-r_{k}(s)\right| \leq K|s-t|^{(1+r) / 2},
$$

which proves (15). Let us finally prove (16). We have by (H2), for a fixed $z$,

$$
\mathbb{E}\left|1_{(Y(t) \leq z)}-1_{(Y(s) \leq z)}\right|^{2} \leq\left|G_{t}(z)-G_{t, s}(z, z)\right|+\left|G_{s}(z)-G_{t, s}(z, z)\right| \leq K|s-t|^{1+r},
$$

for some constant $K$. Then by the $c_{2}$-inequality,

$$
\mathbb{E}\left|1_{(Y(t) \leq Z(t))}-1_{(Y(s) \leq Z(s))}\right|^{2} \leq 2 \mathbb{E}\left|1_{(Y(t) \leq Z(t))}-1_{(Y(s) \leq Z(t))}\right|^{2}+2 \mathbb{E}\left|1_{(Y(s) \leq Z(t))}-1_{(Y(s) \leq Z(s))}\right|^{2},
$$

with

$$
\mathbb{E}\left|1_{(Y(t) \leq Z(t))}-1_{(Y(s) \leq Z(t))}\right|^{2} \leq\left|G_{t}(Z(t))-G_{t, s}(Z(t), Z(t))\right|+\left|G_{s}(Z(t))-G_{s}(Z(s) \wedge Z(t))\right| \leq K|s-t|^{1+r},
$$


and

$$
\mathbb{E}\left|1_{(Y(s) \leq Z(t))}-1_{(Y(s) \leq Z(s))}\right|^{2}=G_{t} Z(s)+G_{s}(Z(t))-2 G_{s}(Z(s) \wedge Z(t)) \leq K|s-t|^{1+r} .
$$

and then (16) holds.

By putting together (14), (15) and (16) and by repeatedly using the $c_{2}$-inequality, we arrive at (21).

Now we have to establish that

$$
\mathbb{E}\left|W_{2}(s)-W_{2}(t)\right|^{2} \leq K|t-s|^{1+r}
$$

Put

$$
W_{2}(t)=(k+1) A(t) B(t) .
$$

with $A(t)=1-G_{t}(Y(t)) / G_{t}(Z(t))^{k}, B(t)=d((Z(t)-Y(t)) / Z(t)) 1_{(Y(t) \leq Z(t))}$. We have by readily check that

$$
|A(t)-A(s)| \leq 2 \beta^{-1}|Y(t)-Y(s)|+M_{0} \beta^{-2}|Z(t)-Z(s)|+\beta^{-2}\left|G_{s}(Z(t))-G_{t}(Z(t))\right|,
$$

Then by (H0)-(H3) and the $c_{2}$-inequality

$$
\mathbb{E}|A(t)-A(s)|^{2} \leq K|t-s|^{1+r},
$$

Next

$$
B(t)=d((Z(t)-Y(t)) / Z(t))^{k}\left(1_{(Y(t) \leq Z(t) \wedge Z(s))}+1_{Z(t) \wedge Z(s) \leq Y(t) \leq Z(t)}\right)=B_{1}(t, s)+B_{2}(t, s),
$$

and

with

$$
B(s)=B_{1}(s, t)+B_{2}(s, t)
$$

$$
B_{1}(t, s)=d((Z(t)-Y(t)) / Z(t))^{k} 1_{(Y(t) \leq Z(t) \wedge Z(s))},
$$

and

$$
B_{2}(t, s)=d((Z(t)-Y(t)) / Z(t))^{k} 1_{(Z(t) \wedge Z(s) \leq Y(t) \leq Z(t))}
$$

Then by (H2)

$$
\mathbb{E} B_{2}(t, s)=G_{t}(t)-G_{t}(Z(t) \wedge Z(s)) \leq K|t-s|^{1+r},
$$

and

$$
\mathbb{E} B_{2}(s, t) \leq K|t-s|^{1+r} .
$$

Next, by putting $C(s, t)=1_{(Z(t) \wedge Z(s) \leq Y(t) \leq Z(t))}$,

$$
\left|B_{1}(t, s)-B_{1}(s, t)\right|=C(s, t) d^{\prime}(D(s, t))\left|\frac{Z(t)-Y(t)}{Z(t)}-\frac{Z(s)-Y(s)}{Z(s)}\right|,
$$

where $D(s, t)$ lies between $((Z(t)-Y(t)) / Z(t)$ and $(Z(s)-Y(s)) / Z(s)$. We finally get

$$
\left|B_{1}(t, s)-B_{1}(t, s)\right| \leq\left(Z_{1}^{-1}+Z_{2} Z_{1}^{-2}\right)|Z(t)-Z(s)|+Z_{1}^{-1}|Y(t)-Y(s)|,
$$

By similar methods, we get

$$
\mathbb{E}\left|B_{1}(t, s)-B_{1}(t, s)\right|^{2} \leq K|t-s|^{1+r} .
$$

By combining all that precedes, we get (27), which together with (21) establishes by the $c_{2}$-inequality

$$
\mathbb{E}|W(s)-W(t)|^{2} \leq K|t-s|^{1+r} .
$$


Now we have to prove that

$$
|\mathbb{E} W(s)-\mathbb{E} W(t)|^{2} \leq K|t-s|^{1+r} .
$$

We only sketch this second part. Let us consider $W_{i}(t), i=1,2$. We have

$$
\mathbb{E} W_{1}(t)=K(t) \int_{0}^{Z(t)} m_{t}(u) \mathrm{d} u .
$$

and

$$
\mathbb{E} W_{2}(t)=(k+1) \int_{0}^{Z(t)}\left(1-G_{t}(u) / G_{t}(Z(t))\right)^{k} d\left(\frac{Z(t)-u}{Z(t)}\right) m_{t}(u) \mathrm{d} u .
$$

By (14),(15) and the decomposition of $\left|\gamma_{t}(t)-\gamma_{s}(s)\right|$ used in (25), we have

$$
|K(t)-K(s)| \leq K|s-t|^{1+r / 2} \text {. }
$$

Furthermore

$$
\int_{0}^{Z(t)} m_{t}(u) \mathrm{d} u-\int_{0}^{Z(s)} m_{s}(u) \mathrm{d} u=\int_{Z(t) \wedge Z(s)}^{Z(t)} m_{t}(u) \mathrm{d} u-\int_{Z(t) \wedge Z(s)}^{Z(s)} m_{s}(u) \mathrm{d} u+\int_{0}^{Z(t) \wedge Z(s)}\left(m_{t}(u)-m_{s}(u)\right) \mathrm{d} u .
$$

We then get

$$
\left|\int_{0}^{Z(t)} m_{t}(u) \mathrm{d} u-\int_{0}^{Z(s)} m_{s}(u) \mathrm{d} u\right| \leq 2 M_{0}|Z(t)-Z(s)|+Z_{2} K|s-t|^{1+r / 2}
$$

Then

$$
\left|\mathbb{E} W_{1}(s)-\mathbb{E} W_{1}(t)\right|^{2} \leq K|s-t|^{1+r} .
$$

Now

$$
\mathbb{E} W_{2}(t)=\int_{0}^{Z(t)} S(t, u) \mathrm{d} u
$$

with

$$
S(t, u)=(k+1)\left(1-G_{t}(u) / G_{t}(Z(t))\right)^{k} d\left(\frac{Z(t)-u}{Z(t)}\right) m_{t}(u) .
$$

Then

$$
\begin{aligned}
\mathbb{E} W_{2}(t)-\mathbb{E} W_{2}(t) & =\int_{0}^{Z(t)} S(t, u) \mathrm{d} u-\int_{0}^{Z(s)} S(s, u) \mathrm{d} u \\
& =\int_{Z(t) \wedge Z(s)}^{Z(t)} S(t, u) \mathrm{d} u-\int_{Z(t) \wedge Z(s)}^{Z(s)} S(s, u) \mathrm{d} u+\int_{0}^{Z(t) \wedge Z(s)}(S(t, u)-S(s, u)) \mathrm{d} u .
\end{aligned}
$$

Since $S(t, u)$ is uniformly bounded, we have

$$
0 \leq\left|\int_{Z(t) \wedge Z(s)}^{Z(t)} S(t, u) \mathrm{d} u-\int_{Z(t) \wedge Z(s)}^{Z(s)} S(s, u) \mathrm{d} u\right| \leq K|s-t|^{1+r / 2} .
$$

Moreover, one easily shows by the (H0)-(H3), with similar techniques used when handling $r_{k}(t)$, that

$$
|S(t, u)-S(t, u)| \leq K|s-t|^{1+r / 2} .
$$

Thus

$$
\left|\mathbb{E} W_{2}(s)-\mathbb{E} W_{2}(t)\right|^{2} \leq K|s-t|^{1+r} .
$$

